Прокудин А. Н., Ковтанюк Л. В., Фирсов С. В.

A. N. Prokudin, L. V. Kovtanyuk, S. V. Firsov

КУСОЧНО-ЛИНЕЙНЫЙ ПОТЕНЦИАЛ ПОЛЗУЧЕСТИ В ДЕФОРМАЦИЯХ ПОЛЗУЧЕСТИ БЫСТРОВРАЩАЮЩЕГОСЯ ЦИЛИНДРА

\title{
PIECEWISE LINEAR POTENTIAL FOR CREEP IN CREEP DEFORMATIONS OF A RAPIDLY ROTATING CYLINDER
}

Прокудин Александр Николаевич - кандидат технических наук, ведущий научный сотрудник Института машиноведения и металлургии Дальневосточного отделения Российской академии наук (Россия, Комсомольск-на-Амуре); 681005, Хабаровский край, г. Комсомольск-на-Амуре, ул. Металлургов, 1. E-mail: sunbeam_85@mail.ru.

Mr. Alexander N. Prokudin - PhD in Engineering, Leading researcher, Institute of Engineering Science and Metallurgy, Far Eastern Branch of the Russian Academy of Sciences (Russia, Komsomolsk-on-Amur); 681005, Khabarovsk territory, Komsomolsk-on-Amur, 1 Metallurgists' str. E-mail: sunbeam_85@mail.ru.

Ковтанюк Лариса Валентиновна - доктор физико-математических наук, заведующая лабораторией необратимого деформирования Института автоматики и процессов управления Дальневосточного отделения Российской академии наук (Россия, Владивосток); 690041, Приморский край, г. Владивосток, ул. Радио, 5; тел.: + 7 (4232) 31-02-14. E-mail: 1k@iacp.dvo.ru.

Ms. Larisa V. Kovtanyuk - Dr. hab. of Physics and Mathematics, Head of the Laboratory of Irreversible Deformation, Institute of Automation and Control Processes, Far Eastern Branch of the Russian Academy of Sciences (Russia, Vladivostok); 690041, Primorsky territory, Vladivostok, 5 Radio str.; tel.: + 7 (4232) 31-02-14. E-mail: 1k@iacp.dvo.ru.

Фирсов Сергей Викторович - аспирант Института машиноведения и металлургии Дальневосточного отделения Российской академии наук (Россия, Комсомольск-на-Амуре); 681005, Хабаровский край, г. Комсомольск-на-Амуре, ул. Металлургов, 1. E-mail: firsov.s.new@yandex.ru.

Mr. Sergey V. Firsov - post-graduate student, Institute of Engineering Science and Metallurgy, Far Eastern Branch of the Russian Academy of Sciences (Russia, Komsomolsk-on-Amur); 681005, Khabarovsk territory, Komsomolsk-on-Amur, 1 Metallurgists' str. E-mail: firsov.s.new@yandex.ru.

Аннотация. В статье рассматривается задача ползучести вращающегося с постоянной скоростью однородного кольцевого цилиндра. Задача решается в рамках теории малых деформаций. Для нахождения начальных условий производится решение данной задачи при упругости. Рассмотрены два случая постановки задачи: цилиндр с жёстким включением и полый цилиндр. Для описания ползучести использовался степенной закон Нортона с непрерывным потенциалом по типу Мизеса и с кусочно-линейным потенциалом. Упругое решение ищется аналитически, для ползучести используются численный метод конечных разностей. Сравнены результаты для непрерывного потенциала ползучести и для кусочно-линейного для двух вариантов граничных условий.

Summary. In article the creep problem of a uniform circular cylinder rotating at a constant speed is considered. The problem is solved within the framework of the theory of small deformations. To find the initial conditions, the solution of the given problem is performed under elasticity. Two cases of statement of the problem are considered: a cylinder with a rigid inclusion and a hollow cylinder. To describe the creep, the Norton power law with a continuous potential of the Mises type and with a piecewise linear potential was used. An elastic solution is sought analytically; a numerical finite difference method is used for creep. The results for a continuous creep potential are also compared for a piecewise-linear boundary condition for two variants.

Ключевые слова: малые деформации, упругость, ползучесть.

Key words: small deformation, elasticity, creep.

УДК 539.374 


\section{Учёные записки}

Комсомольского-на-Амуре государственного технического университета

Работа выполнена в рамках государственного задания № 007-00285-18-00.

\section{Введение}

Вращающиеся цилиндры и диски широко применяются в авиастроении, авиакосмической промышленности, ядерной энергетике. Деформирование цилиндров происходит под действием центробежных сил, но также могут иметь место внешние нагрузки, температурные и электромагнитные поля. Исследование механического поведения вращающихся цилиндров для различных типов материалов представляет значительный интерес, ежегодно публикуется большое количество работ по этой теме. При этом чаще всего используется квазистатическая постановка и предположение о плоском деформированном состоянии в цилиндре. В простейшем случае материал цилиндра является линейно-упругим телом. Решение такой задачи входит во многие книги по теории упругости [6; 9; 19]. В работе [7] приведено универсальное решение для нелинейно упругого материала, в котором вид потенциальной энергии может быть произвольным. Подробно поведение гиперупругих вращающихся цилиндров для различных упругих потенциалов и граничных условий было исследовано в работах $[11 ; 16]$. Когда скорость вращения достигает критического значения, в цилиндре начинает развиваться пластическое течение.

На долговременную прочность вращающихся цилиндров существенное влияние оказывает ползучесть материалов. В известной монографии Ю. Н. Работнова [8] приведено решение о вращающемся диске и цилиндре с использованием экспоненциального закона ползучести. В работах $[12 ; 13 ; 14]$ исследовалась ползучесть ортотропных цилиндров на основе степенного закона ползучести Нортона. Большое внимание уделяется явлению ползучести функционально-градиентных материалов (см. публикации [15; 17]).

Целью данной работы является исследование ползучести однородного вращающегося цилиндра с помощью кусочно-линейного степенного потенциала. Использование кусочно-линейных потенциалов является классическим подходом при решении задач теории пластичности. К таким потенциалам относятся условия пластичности Треска - Сен-Венана, Ишлинского - Ивлева, Кулона - Мора и другие. В работах [4; 5] Д. Д. Ивлев высказал идею использовать кусочно-линейные потенциалы в гидродинамике вязкой жидкости и нелинейной теории упругости. Позднее Г. И. Быковцев предложил использовать кусочно-линейные потенциалы для математического описания явления ползучести [2; 3]. Именно использование кусочно-линейного потенциала ползучести позволило получить точное решение задачи ползучести и релаксации напряжений в бесконечной пластине с первоначально круглым отверстием [1]. Данная публикация продолжает исследования, изложенные в работах [2; 3].

\section{Постановка задачи}

Рассмотрим кольцевой цилиндр с внутренним радиусом $R_{1}$ и внешним $R_{2}$. Цилиндр может иметь жёсткое включение в центре либо быть полым.

Цилиндр вращается с угловой скоростью $\omega(t)$. Считаем, что угловое ускорение мало. Введём цилиндрическую систему координат $r, \theta, z$. Сила, действующая на диск, имеет только радиальную компоненту и равна $F_{r}=\rho r \omega^{2}$, где $\rho$ - плотность материала. Примем, что деформации, возникающие в цилиндре, являются малыми. Тензор малых деформаций имеет следующий общий вид:

$$
E=\frac{1}{2}\left(\nabla_{X} \boldsymbol{u}+\left(\nabla_{X} \boldsymbol{u}\right)^{T}\right)
$$

где $\nabla_{X}$ - оператор градиента; $\boldsymbol{u}$ - вектор перемещения $\boldsymbol{u}=u_{r} \mathbf{e}_{r}+u_{\theta} \mathbf{e}_{\theta}+u_{z} \boldsymbol{e}_{z}$.

Полные деформации $d_{i j}$ равны сумме упругих деформаций $e_{i j}$ и деформаций ползучести $p_{i j}$. В условиях осевой симметрии и плоского деформированного состояния имеем 


$$
\begin{gathered}
u_{r}=u_{r}(r), u_{\theta}=0, u_{z}=0 ; \\
e_{r r}+p_{r r}=d_{r r}=\frac{\partial u_{r}}{\partial r} ; \\
e_{\theta \theta}+p_{\theta \theta}=d_{\theta \theta}=\frac{u_{r}}{r} ; \\
e_{z z}+p_{z z}=d_{z z}=0,
\end{gathered}
$$

где запятая в нижнем индексе обозначает производную по координате. Напряжения выражаются через упругие деформации с помощью закона Гука:

$$
\begin{gathered}
\sigma_{r r}=\frac{E}{(1+v)(1-2 v)}\left((1-v)\left(\frac{\partial u_{r}}{\partial r}-p_{r r}\right)+v\left(\frac{u_{r}}{r}-p_{\theta \theta}\right)-v p_{z z}\right), \\
\sigma_{\theta \theta}=\frac{E}{(1+v)(1-2 v)}\left(v\left(\frac{\partial u_{r}}{\partial r}-p_{r r}\right)+(1-v)\left(\frac{u_{r}}{r}-p_{\theta \theta}\right)-v p_{z z}\right), \\
\sigma_{z z}=\frac{E}{(1+v)(1-2 v)}\left(v\left(\frac{\partial u_{r}}{\partial r}-p_{r r}\right)+v\left(\frac{u_{r}}{r}-p_{\theta \theta}\right)-(1-v) p_{z z}\right),
\end{gathered}
$$

где $E$ - модуль Юнга; $v$ - коэффициент Пуассона.

С учётом вышеприведённых допущений останется только одно нетривиальное уравнение равновесия в перемещениях:

$$
\begin{gathered}
\frac{\partial^{2} u_{r}}{\partial r^{2}}+\frac{1}{r} \frac{\partial u_{r}}{\partial r}-\frac{1}{r^{2}} u_{r}=-\frac{F_{r}}{A}+ \\
+\frac{\partial p_{r r}}{\partial r}+\frac{v}{(1-v)}\left(\frac{\partial p_{\theta \theta}}{\partial r}+\frac{\partial p_{z z}}{\partial r}\right)+\frac{(1-2 v)}{(1-v)}\left(\frac{p_{r r}}{r}-\frac{p_{\theta \theta}}{r}\right) ; A=\frac{E(1-v)}{(1+v)(1-2 v) .}
\end{gathered}
$$

Видно, что если деформации ползучести отсутствуют, то предыдущее уравнение сводится к уравнению Навье. Скорости деформации ползучести определяются следующим законом: $\dot{p}_{i j}=\frac{\partial C}{\partial \sigma_{i j}}$, где $C$ - потенциал ползучести. Запишем потенциал $C$ в соответствии со степенным законом ползучести Нортона:

$$
C=B \Sigma^{n},
$$

где $B, n$ - параметры материала, определяемые экспериментально; $\Sigma$ - эквивалентное напряжение.

Обычно $\Sigma$ выбирается в виде эквивалентного напряжения по Мизесу: $\Sigma_{1}=\sqrt{\frac{3}{2}} \cdot \sqrt{\left(\sigma_{1}-\sigma\right)^{2}+\left(\sigma_{2}-\sigma\right)^{2}+\left(\sigma_{3}-\sigma\right)^{2}}$, где $\sigma_{1}, \sigma_{2}, \sigma_{3}$ - главные напряжения; $\sigma=\frac{1}{3}\left(\sigma_{1}+\sigma_{2}+\sigma_{3}\right)$ гидростатическое напряжение.

Поскольку в нашем случае в тензоре напряжений присутствуют только диагональные компоненты, предыдущее выражение можно преобразовать к виду

$$
\Sigma_{1}=\sqrt{\sigma_{r r}^{2}+\sigma_{\theta \theta}^{2}+\sigma_{z z}^{2}-\sigma_{r r} \sigma_{\theta \theta}-\sigma_{\theta \theta} \sigma_{z z}-\sigma_{r r} \sigma_{z z}}
$$


Кусочно-линейный потенциал ползучести, для которого эквивалентное напряжение $\Sigma_{2}=\max \left|\sigma_{i}-\sigma_{j}\right|$, предложил использовать Г. И. Быковцев [2; 3]. Потенциалы в формуле (6), соответствующие $\Sigma_{1}$ и $\Sigma_{2}$, в дальнейшем будем обозначать как $C_{1}$ и $C_{2}$. Для потенциала $C_{1}$ можно получить явное выражение для скоростей деформаций ползучести:

$$
\begin{aligned}
& \dot{p}_{r r}=\frac{B n \Sigma_{1}^{n-2}}{2}\left(2 \sigma_{r r}-\sigma_{\theta \theta}-\sigma_{z z}\right), \\
& \dot{p}_{\theta \theta}=\frac{B n \Sigma_{1}^{n-2}}{2}\left(2 \sigma_{\theta \theta}-\sigma_{r r}-\sigma_{z z}\right), \\
& \dot{p}_{z z}=\frac{B n \Sigma_{1}^{n-2}}{2}\left(2 \sigma_{z z}-\sigma_{r r}-\sigma_{\theta \theta}\right) .
\end{aligned}
$$

Для потенциала $C_{2}$ уравнения для скоростей пластических деформаций будут иметь разный вид в зависимости от соотношения между величинами напряжений $\sigma_{r r}, \sigma_{\theta \theta}, \sigma_{z z}$. Уравнения в главных напряжениях имеют следующий вид:

Если $\sigma_{1}>\sigma_{2}>\sigma_{3}$, то

$$
\dot{p}_{1}=B n \Sigma_{1}^{n-1} ; \dot{p}_{2}=0 ; \dot{p}_{3}=-B n \Sigma_{1}^{n-1} \text {. }
$$

Если $\sigma_{1}=\sigma_{2}>\sigma_{3}$, то

$$
\dot{p}_{1}=\frac{B n \Sigma_{1}^{n-1}}{2} ; \dot{p}_{2}=\frac{B n \Sigma_{1}^{n-1}}{2} ; \dot{p}_{3}=-B n \Sigma_{1}^{n-1}
$$

Если $\sigma_{1}>\sigma_{2}=\sigma_{3}$, то

$$
\dot{p}_{1}=B n \Sigma_{1}^{n-1} ; \dot{p}_{2}=-\frac{B n \Sigma_{1}^{n-1}}{2} ; \dot{p}_{3}=-\frac{B n \Sigma_{1}^{n-1}}{2} .
$$

Начальные и граничные условия задачи. В начальный момент времени перемещения, напряжения и необратимые деформации в среде отсутствуют. Если цилиндр имеет жёсткое включение в центре, то тогда на внутренней поверхности цилиндра $r=R_{1}$ задано перемещение, а на внешней $r=R_{2}$ - напряжение:

$$
u_{r}\left(R_{1}\right)=0, \sigma_{r r}\left(R_{2}\right)=0 \text {. }
$$

Для полого цилиндра и на внутренней и внешней поверхностях задаются напряжения:

$$
\sigma_{r r}\left(R_{1}\right)=0, \sigma_{r r}\left(R_{2}\right)=0 .
$$

\section{Упругое решение}

Если в цилиндре отсутствуют деформации ползучести, то тогда уравнение (4) сводится к уравнению Навье, его общее решение имеет вид

$$
u_{r}=\frac{C_{1}}{r}+C_{2} r-\frac{1}{8} \frac{\rho r^{3} \omega^{2}}{A}
$$


При этом значения постоянных $C_{1}$ и $C_{2}$ для цилиндра с жёстким включением имеют вид

$$
C_{1}=-\frac{\rho R_{1}^{2} R_{2}^{2}\left(R_{1}^{2}+(2 v-3) R_{2}^{2}\right) \omega^{2}}{8 A\left((2 v-1) R_{1}^{2}-R_{2}^{2}\right)}, C_{2}=\frac{\rho\left((2 v-1) R_{1}^{4}+(2 v-3) R_{2}^{4}\right) \omega^{2}}{8 A\left((2 v-1) R_{1}^{2}-R_{2}^{2}\right)} \text {. }
$$

Для полого цилиндра $C_{1}$ и $C_{2}$ имеют такой вид:

$$
C_{1}=\frac{p(2 v-3) R_{1}^{2} R_{2}^{2} \omega^{2}}{8 A(2 v-1)} ; C_{2}=-\frac{p(2 v-3)\left(R_{1}^{2}+R_{2}^{2}\right) \omega^{2}}{8 A} \text {. }
$$

\section{Численная схема}

Для численного решения уравнения (5) воспользуемся методом конечных разностей. Построим в области деформирования $\left(R_{1}, R_{2}\right)$ равномерную сетку из $k$-узлов с координатами $r_{0}, r_{1}, \ldots, r_{k-1}$. Узел с номером 0 соответствует левой границе области, а с номером $k-1-$ правой границе. Шаг сетки обозначим как $\Delta r$.

Выражение для $\Delta r$ имеет вид

$$
\Delta r=\frac{R_{2}-R_{1}}{n-1}
$$

Координату і-го узла можно найти следующим образом:

$$
r_{i}=R_{1}+i \cdot \Delta r .
$$

Процесс деформирования разобьём на $l$ временных шагов продолжительности $\Delta t$.

Значение некоторой неизвестной функции в узловых точках будем обозначать как $y^{i, j}$, где $i$ - номер узла, а $j$ - номер временного шага. Считаем, что шаг $j=0$ соответствует упругому решению. Для аппроксимации пространственных переменных будем применять центральные шаблоны аппроксимации 2-го порядка точности:

$$
\frac{\partial^{2} y}{\partial r^{2}} \cong \frac{y^{i-1, j}-2 y^{i, j}+y^{i+1, j}}{\Delta r^{2}}, i=1 . . k-2, j=1 . . l ; \frac{\partial y}{\partial r} \cong \frac{y^{i+1, j}-y^{i-1, j}}{2 \Delta r}, i=1 . . k-2, j=1 . . l .
$$

\section{Результаты расчётов}

Рассмотрим следующие значения параметров задачи:

$R_{1}=0,02 \mu, R_{2}=0,1 \mathcal{M} ; \quad E=210 \cdot 10^{9} \Pi a, v=0,38 ; \quad B=4,1 \cdot 10^{-22}, n=3 ; \omega=1600 ; \rho=7,7 \cdot 10^{3} \kappa 2 / \mathcal{M}^{3} ;$ $k=1000, l=8000, \Delta t=0,1 c$.

Перейдём к рассмотрению процесса ползучести в цилиндре. Для удобства будем оценивать необратимые деформации с помощью следующего инварианта тензора деформаций ползучести:

$$
P_{2}=\sqrt{p_{r r}^{2}+p_{\theta \theta}^{2}+p_{z z}^{2}-p_{r r} p_{\theta \theta}-p_{\theta \theta} p_{z z}-p_{r r} p_{z z}} .
$$

На рис. 1 показаны перемещения в цилиндре с жёстким включением для моментов времени $\mathrm{t}=0,400,800$ с. Видим, что для потенциалов $C_{1}$ и $C_{2}$ получены близкие результаты. 
a)

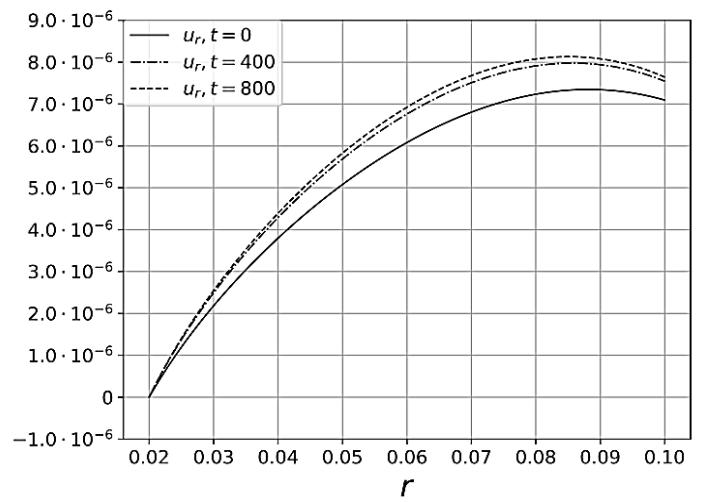

б)

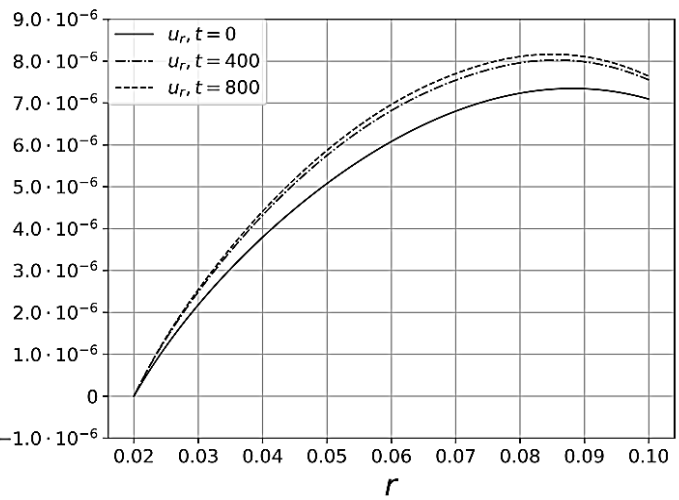

Рис. 1. Перемещения в цилиндре с жёстким включением с учётом ползучести материала: а - потенциал $C_{1} ; \sigma$ - потенциал $C_{2}$

Иная картина (см. рис. 2) наблюдается в полом цилиндре: использование кусочнолинейного потенциала $C_{2}$ приводит к существенному увеличению перемещений.

a)

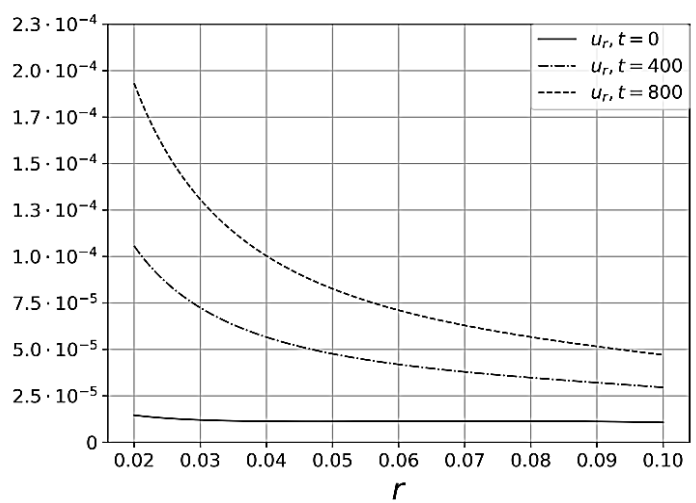

б)

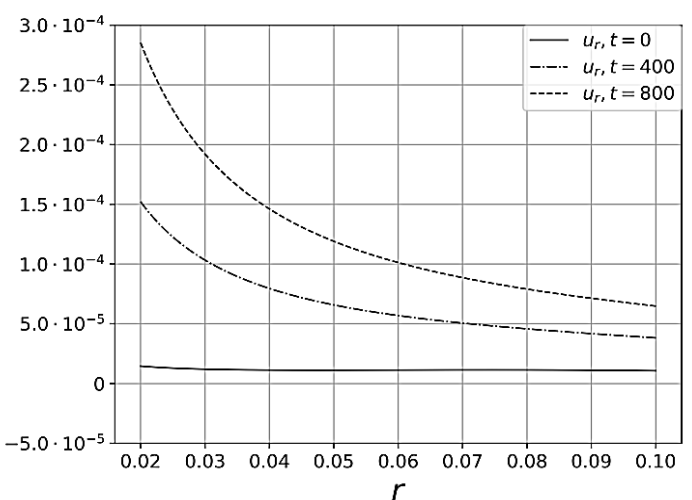

Рис. 2. Перемещения в полом цилиндре с учётом ползучести материала: а - потенциал $C_{1} ;$ б - потенциал $C_{2}$

Аналогичная закономерность наблюдается и для необратимых деформаций (см. рис. 3,4 ).

a)

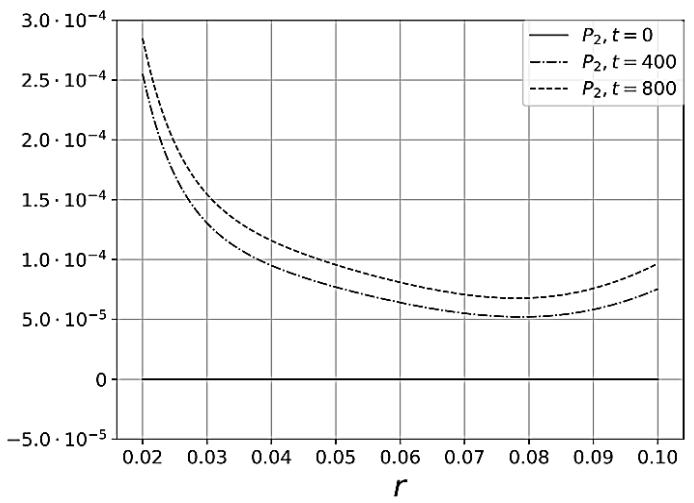

б)

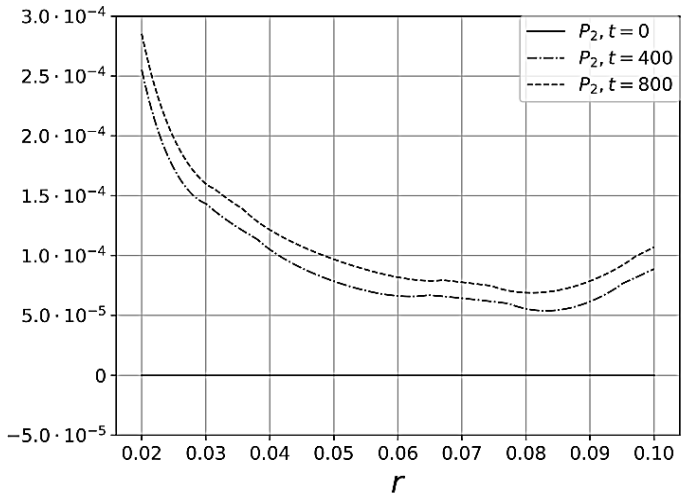

Рис. 3. Инвариант $P_{2}$ в цилиндре с жёстким включением с учётом ползучести материала: а - потенциал $C_{1} ;$ б- потенциал $C_{2}$ 
Прокудин А. Н., Ковтанюк Л. В., Фирсов С. В.

КУСОЧНО-ЛИНЕЙНЫЙ ПОТЕНЦИАЛ ПОЛЗУЧЕСТИ В ДЕФОРМАЦИЯХ ПОЛЗУЧЕСТИ БЫСТРОВРАЩАЮЩЕГОСЯ ЦИЛИНДРА

a)

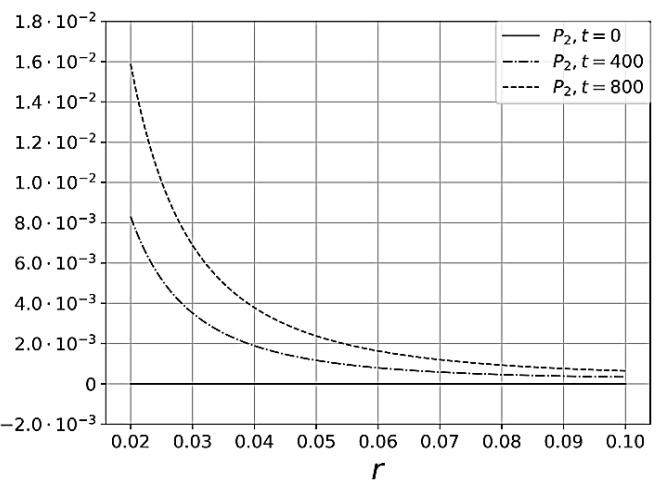

б)

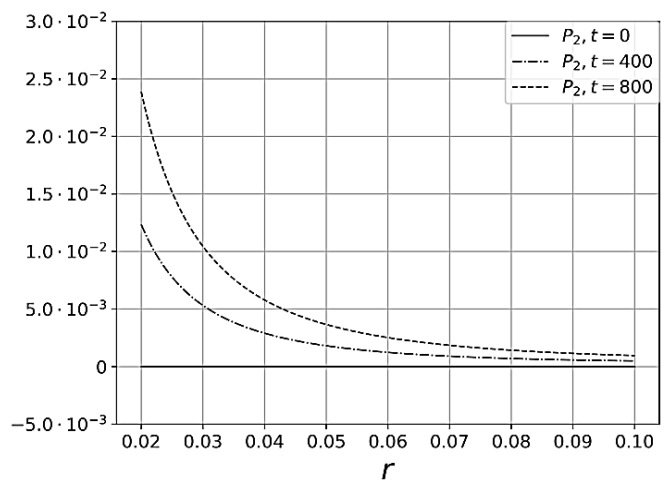

Рис. 4. Инвариант $P_{2}$ в полом цилиндре с учётом ползучести материала:

$$
\text { а - потенциал } C_{1} ; \text { б - потенциал } C_{2}
$$

Оценим напряжения, возникающие в ходе ползучести материала. На рис. 5 и 6 представлены графики инварианта $\Sigma_{1}$. Напряжения в цилиндре слабо зависят от типа используемого потенциала $C_{1}$ или $C_{2}$ и со временем снижаются. Это справедливо и для цилиндра с жёстким включением, и для полого цилиндра.

a)

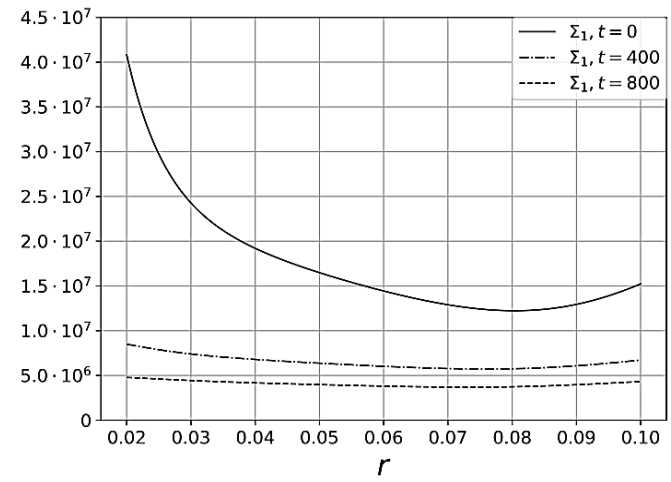

б)

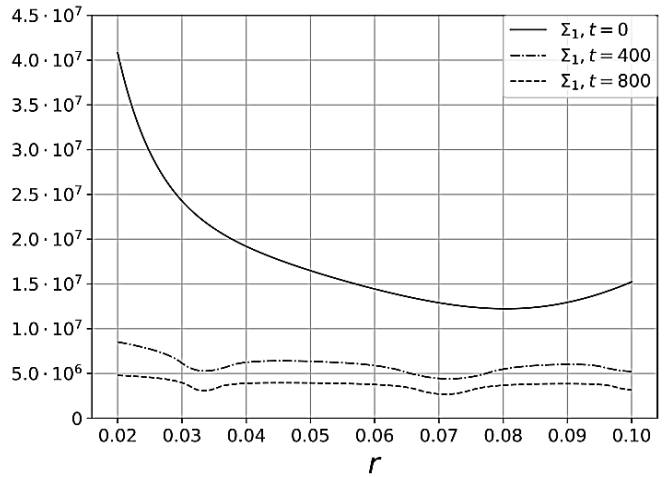

Рис. 5. Напряжения $\sum_{1}$ в цилиндре с жёстким включением с учётом ползучести материала: а - потенциал $C_{1} ;$ б- потенциал $C_{2}$

a)

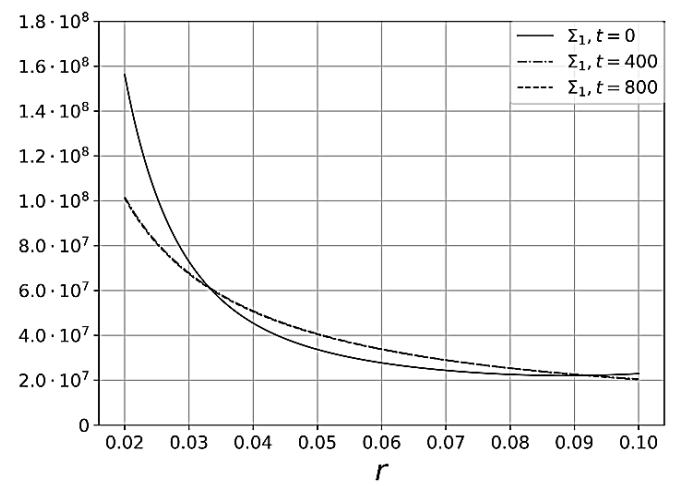

б)

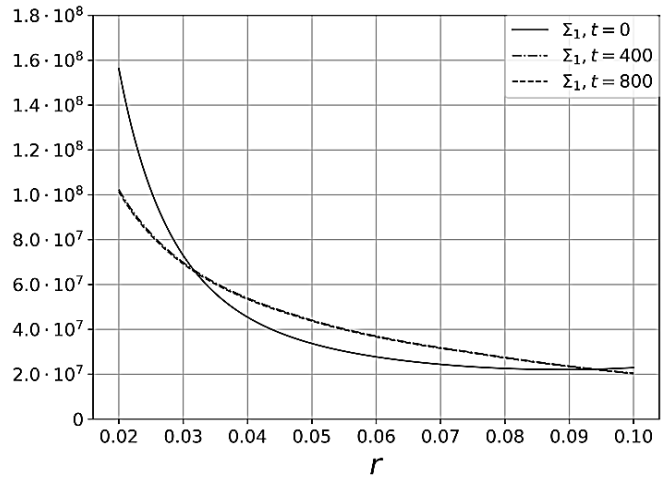

Рис. 6. Напряжения $\sum_{1}$ в полом цилиндре с учётом ползучести материала: а - потенциал $C_{1} ; \sigma$ - потенциал $C_{2}$ 


\section{Учёные записки}

Комсомольского-на-Амуре государственного технического университета

ЛИТЕРАТУРА

1. Буренин, А. А. Плоское напряжённое состояние в условиях нелинейной неустановившейся ползучести / А. А. Буренин, В. М. Ярушина // Дальневосточный математический журнал. Владивосток: Институт прикладной математики Дальневосточного отделения академии наук. - 2002. - Т. 3. - № 1. - С. 64-78.

2. Быковцев, Г. И. Кусочно-линейные потенциалы в нелинейной механике / Г. И. Быковцев, Н. Г. Быковцева // Доклады Академии наук. - 1994. - Т. 335. - № 3. - С. 310-312.

3. Быковцев, Г. И. Об особенностях модели неустановившейся ползучести, основанной на использовании кусочно-линейных потенциалов / Г. И. Быковцев, В. М. Ярушина // Проблемы механики сплошных сред и элементов конструкций: сборник научных трудов. - Владивосток: Дальнаука, 1998. - С. 9-26.

4. Ивлев, Д. Д. К построению гидродинамики вязкой жидкости / Д. Д. Ивлев // Доклады Академии наук СССР. 1960. - Т. 135. - № 2. - С. 280-282.

5. Ивлев, Д. Д. Построение теории упругости / Д. Д. Ивлев // Доклады Академии наук СССР. - 1961. Т. 138. - № 6. - С. 1321-1324.

6. Ландау, Л. Д. Теоретическая физика. В 10 т. Т. 7. Теория упругости: учеб. пособие для вузов Л. Д. Ландау, Е. М. Лифшиц. - М.: ФИЗМАТЛИТ, 2003. - 264 с.

7. Лурье, А. И. Нелинейная теория упругости / А. И. Лурье. - М.: Наука, 1980. - 512 с.

8. Работнов, Ю. Н. Ползучесть элементов конструкций / Ю. Н. Работнов. - М.: Наука, 1966. - 752 с.

9. Тимошенко, С. П. Теория упругости / С. П. Тимошенко, Дж. Гудьер. - М.: Наука, 1979. - 576 с.

10. Arslan, E. The rotating elastic-plastic hollow shaft conveying a hot medium / E. Arslan, W. Mack, A. N. Eraslan // Forsch Ingenieurwes. - 2010. - Vol. 74. - № 1. - P. 27-39.

11. Bagheri, A. On the behavior of rotating thick-walled cylinders made of hyperelastic materials / A. Bagheri, D. Taghizadeh, H. Darijani // Meccanica. - 2016. - Vol. 51. - № 3. - P. 673-692.

12. Bhatnaga, N. S. Creep Analysis of Orthotropic Rotating Cylinder / N. S. Bhatnaga, V. K. Arya, K. K. Debnath // J. Pressure Vessel Technol. - 1980. - Vol. 102. - № 4. - P. 371-377.

13. Bhatnagar, N. S. Creep analysis of an internally pressurised orthotropic rotating cylinder / N. S. Bhatnagar, P. S. Kulkarni, V. K Arya // Nuclear Engineering and Design. - 1984. - Vol. 83. - № 3. - P. 379-388.

14. Bhatnagar, N. S. Creep analysis of orthotropic rotating cylinders considering finite strains / N. S. Bhatnagar, P. S. Kulkarni, V. K. Arya // International Journal of Non-Linear Mechanics. - 1986. - Vol. 21. - № 1. - P. 61-71. 15. Bose, T. Effect of thermal gradation on steady state creep of functionally graded rotating disc / T. Bose, M. Rat$\tan / /$ European Journal of Mechanics - A/Solids. - 2018. - Vol. 67. - P. 169-176.

16. Chadwick, P. The deformation of rubber cylinders and tubes by rotation / P. Chadwick, C. F. M. Creasy, V. G. Hart // The ANZIAM Journal. - 1977. - Vol. 20. - № 1. - P. 62-96.

17. Mangal, S. K. Steady-State Creep Analysis of Functionally Graded Rotating Cylinder / S. K. Mangal, N. Kapoor, T. Singh // Strain. - 2013. - Vol. 49. - № 6. - P. 457-466.

18. Nadai, A. Theory of Flow and Fracture of Solids / A. Nadai. - McGraw Hill, 1950. - Volume One. - 572 p. 19. Sadd, M. H. Elasticity: theory, applications, and numerics. Third edition / M. H. Sadd. - Amsterdam; Boston: Elsevier/AP, Academic Press is an imprint of Elsevier, 2014. - 582 p. 